\title{
Dynamic Portfolio Selection Under Ambiguity in the $\epsilon$-Contaminated Binomial Model
}

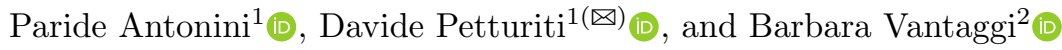 \\ 1 Dip. Economia, University of Perugia, Perugia, Italy \\ paride.antonini@studenti.unipg.it, davide.petturiti@unipg.it \\ 2 Dip. MEMOTEF, "La Sapienza" University of Rome, Rome, Italy \\ barbara.vantaggi@uniroma1.it
}

\begin{abstract}
Investors often need to look for an optimal portfolio acting under ambiguity, as they may not be able to single out a unique realworld probability measure. In this paper a discrete-time dynamic portfolio selection problem is studied, referring to an $\epsilon$-contaminated binomial market model and assuming investors' preferences are consistent with the Choquet expected utility theory. We formulate the portfolio selection problem for a CRRA utility function in terms of the terminal wealth, and provide a characterization of the optimal solution in the case stock price returns are uniformly distributed. In this case, we further investigate the effect of the contamination parameter $\epsilon$ on the optimal portfolio.
\end{abstract}

Keywords: Ambiguity $\cdot$ Optimal portfolio $\cdot \epsilon$-Contamination model Choquet integral

\section{Introduction}

The standard approach to uncertainty in portfolio selection models is to refer to a unique real-world probability measure $\mathbf{P}$, usually estimated from data. Nevertheless, sometimes, due to the presence of unobserved variables or since the information is partial, misspecified or "imprecise", it is no longer possible to handle uncertainty through a single probability measure. This has led to the development of models taking care of ambiguity, i.e., coping with a class of probability measures.

In recent years a growing interest has been addressed towards ambiguity in decision and economic literature (see, e.g., the survey papers [5] and [6]). Mainly, the aim is to propose models generalizing the classical subjective expected utility model in a way to avoid paradoxical situations like that discussed in [4], showing preference patterns under ambiguity that are not consistent with the expected utility paradigm. In particular, referring to the Anscombe-Aumann framework, the seminal papers [17] and [7] incorporate ambiguity in decisions, respectively, either through a non-additive uncertainty measure or through a class of probability measures. The two approaches are generally not equivalent but in case 
of a 2-monotone capacity [19] they reduce to Choquet expected utility. Choquet expected utility has been investigated also in a von Neumann-Morgenstern-like framework, by referring to "objective" completely monotone capacities modeling generalized lotteries $[2,10,14]$.

In this paper, we formalize a dynamic portfolio selection problem under ambiguity, referring to an $\epsilon$-contamination [9] of a binomial market model [3] and to a CRRA utility function [18]. Thanks to the completeness of the market, we formulate the dynamic portfolio selection in terms of the final wealth. Moreover, since the lower envelope of the $\epsilon$-contamination class, obtained from the real-world probability $\mathbf{P}$, is a completely monotone capacity, we assume that the investors' preferences are consistent with the Choquet expected utility theory. The problem amounts to finding a final wealth maximizing the corresponding Choquet expected utility functional. We stress that, due to the properties of the Choquet integral [8], an agent which is a Choquet expected utility maximizer is actually an expected utility maximinimizer, with respect to the $\epsilon$-contamination class.

Notice that, referring to a one-period model, a portfolio selection problem under ambiguity via maximinimization has been faced in $[15,16]$. Moreover, our problem is analogous to that formulated in Appendix $\mathrm{C}$ of [11], the latter differing from ours for working in a continuous setting and considering a distortion of the probability $\mathbf{P}$.

We show that our problem can be reduced to a family of linearly constrained concave problems, indexed by the set of all permutations of the sample space. Moreover, the initial problem is proved to have a unique optimal solution. Focusing on the special case of uniformly distributed stock returns (determining a uniform probability distribution on the sample space) we provide a characterization of the optimal solution relying only on a number of permutations equal to the cardinality of the sample space. Yet in the uniform case, we study the effect of the contamination parameter $\epsilon$ on the optimal portfolio, showing the presence of a threshold above which the optimal self-financing strategy reduces to 0 for all times: this highlights that with such values of $\epsilon$ the ambiguity in the model is so high to make the risk-free portfolio the most suitable choice.

This paper differs from the approach used in [12], where among their results, the authors characterize a generalization of the random walk by replacing a probability with a capacity on the branches of a tree, for each time and by imposing dynamic consistency (or rectangularity). We stress that dynamic consistency is a mathematically helpful property in order to capture a form of martingale property, but it is a strong limitation for a non-additive measure.

\section{Dynamic Portfolio Selection in the Binomial Model}

We refer to the multi-period binomial model introduced by Cox, Ross and Rubinstein [3]. Such model considers a perfect (competitive and frictionless) market under no-arbitrage, where two basic securities are traded: a non-dividend-paying stock and a risk-free bond. 
The evolution of the prices of such securities is expressed by the stochastic processes $\left\{S_{0}, \ldots, S_{T}\right\}$ and $\left\{B_{0}, \ldots, B_{T}\right\}$, with $T \in \mathbb{N}, S_{0}=s>0, B_{0}=1$, and for $t=1, \ldots, T$,

$$
\frac{S_{t}}{S_{t-1}}=\left\{\begin{array}{l}
u, \text { with probability } p \\
d, \text { with probability } 1-p
\end{array} \text { and } \frac{B_{t}}{B_{t-1}}=(1+r),\right.
$$

where $u>d>0$ are the "up" and "down" stock price coefficients, $r$ is the risk-free interest rate over each period, satisfying $u>(1+r)>d$, and $p \in(0,1)$ is the probability of an "up" movement for the stock price.

All the processes we consider are defined on a filtered probability space $\left(\Omega, \mathcal{F},\left\{\mathcal{F}_{t}\right\}_{t=0, \ldots, T}, \mathbf{P}\right)$, where $\Omega=\left\{1, \ldots, 2^{T}\right\}$ and $\mathcal{F}_{t}$ is the algebra generated by random variables $\left\{S_{0}, \ldots, S_{t}\right\}$, for $t=0, \ldots, T$, with $\mathcal{F}_{0}=\{\emptyset, \Omega\}$ and $\mathcal{F}_{T}=\mathcal{F}=\wp(\Omega)$, where the latter denotes the power set of $\Omega$. As usual $\mathbf{E}^{\mathbf{P}}$ denotes the expected value with respect to $\mathbf{P}$.

The process $\left\{S_{0}, \ldots, S_{T}\right\}$ is a multiplicative binomial process where the returns $\frac{S_{1}}{S_{0}}, \ldots, \frac{S_{T}}{S_{T-1}}$ are i.i.d. random variables, and the probability $\mathbf{P}$, usually said real-world probability, is completely singled out by the parameter $p$, indeed, for $t=0, \ldots, T$ and $k=0, \ldots, t$, we have that

$$
\mathbf{P}\left(S_{t}=u^{k} d^{t-k} s\right)=\frac{t !}{k !(t-k) !} p^{k}(1-p)^{t-k}
$$

We consider a self-financing strategy $\left\{\theta_{0}, \ldots, \theta_{T-1}\right\}$, that is an adapted process where each $\theta_{t}$ is the (random) number of shares of stock to buy (if positive) or short-sell (if negative) at time $t$ up to time $t+1$. Such strategy determines an adapted wealth process $\left\{V_{0}, \ldots, V_{T}\right\}$, where, for $t=0, \ldots, T-1$,

$$
V_{t+1}=(1+r) V_{t}+\theta_{t} S_{t}\left(\frac{S_{t+1}}{S_{t}}-(1+r)\right) .
$$

This market model is said to be complete, i.e., there is a unique probability measure $\mathbf{Q}$ on $\mathcal{F}$ equivalent to $\mathbf{P}$, usually said risk-neutral probability, such that the discounted wealth process of any self-financing strategy is a martingale under $\mathbf{Q}$ :

$$
\frac{V_{t}}{(1+r)^{t}}=\mathbf{E}_{t}^{\mathbf{Q}}\left[\frac{V_{T}}{(1+r)^{T}}\right]
$$

for $t=0, \ldots, T$, where $\mathbf{E}_{t}^{\mathbf{Q}}[\cdot]=\mathbf{E}^{\mathbf{Q}}\left[\cdot \mid \mathcal{F}_{t}\right]$ and $\mathbf{E}^{\mathbf{Q}}$ is the expectation with respect to $\mathbf{Q}$. Notice that $\mathbf{E}_{0}^{\mathbf{Q}}$ can be identified with $\mathbf{E}^{\mathbf{Q}}$. In particular, completeness implies that every payoff $V_{T} \in \mathbb{R}^{\Omega}$ depending only on the stock price history can be replicated by a dynamic self-financing strategy $\left\{\theta_{0}, \ldots, \theta_{T-1}\right\}$ and its unique no-arbitrage price at time $t=0$ is

$$
V_{0}=\frac{\mathbf{E}^{\mathbf{Q}}\left[V_{T}\right]}{(1+r)^{T}}
$$


Notice that the process $\left\{S_{0}, \ldots, S_{T}\right\}$ continues to be a multiplicative binomial process also under $\mathbf{Q}$, the latter being completely characterized by the parameter

$$
q=\frac{(1+r)-d}{u-d} \in(0,1)
$$

which replaces $p$. In particular, every state $i \in \Omega$ is identified with the path of the stock price evolution corresponding to the $T$-digit binary expansion of number $i-1$, in which zeroes are interpreted as "up" movements and ones as "down" movements. If in such representation there are $k$ "up" movements and $T-k$ "down" movements, then (we avoid braces to simplify writing)

$$
\mathbf{P}(i)=p^{k}(1-p)^{T-k} \quad \text { and } \quad \mathbf{Q}(i)=q^{k}(1-q)^{T-k} .
$$

In the market model we have just introduced, the real-world probability measure $\mathbf{P}$ is assumed to encode the beliefs of an investor. Assuming that investor's preferences are consistent with the expected utility theory, given a utility function $u$ with $\operatorname{dom} u=\mathbb{R}_{++}=(0,+\infty)$, usually assumed to be sufficiently regular, strictly increasing and strictly concave, and an initial wealth $V_{0}>0$, the dynamic portfolio selection consists in determining a self-financing strategy $\left\{\theta_{0}, \ldots, \theta_{T-1}\right\}$ resulting in a final wealth $V_{T} \in \mathbb{R}_{++}^{\Omega}$ solving

$$
\max _{\theta_{0}, \ldots, \theta_{T-1}} \mathbf{E}^{\mathbf{P}}\left[u\left(V_{T}\right)\right] .
$$

Due to the dynamic consistency property of the conditional expected value operator, this problem can be efficiently solved through dynamic programming [18].

In this paper we suppose that our investor has ambiguous beliefs, meaning that, rather than having a single probability $\mathbf{P}$, he/she actually considers a class of probabilities $\mathcal{P}$. In this case, the choice of $\left\{\theta_{0}, \ldots, \theta_{T-1}\right\}$ should take into account all the probabilities in $\mathcal{P}$, by adopting a suitable criterion of choice, justified by a normative criterion of rationality.

\section{Modeling Ambiguity Through the $\epsilon$-Contamination Model}

Here we consider the binomial market model (see Sect. 2), introducing ambiguity to get an $\epsilon$-contaminated binomial model. Given the real-world probability $\mathbf{P}$ defined on $\mathcal{F}$ (which is completely singled out by $p$ ) and $\epsilon \in(0,1)$, the corresponding $\epsilon$-contamination model (see, e.g., $[9,19]$ ) is the class of probability measures on $\mathcal{F}$ defined as

$$
\mathcal{P}_{p, \epsilon}=\left\{\mathbf{P}^{\prime}=(1-\epsilon) \mathbf{P}+\epsilon \mathbf{P}^{\prime \prime}: \mathbf{P}^{\prime \prime} \text { is a probability measure on } \mathcal{F}\right\}
$$

whose lower envelope $\nu_{p, \epsilon}=\min \mathcal{P}_{p, \epsilon}$ is a completely monotone normalized capacity defined on $\mathcal{F}$ such that

$$
\nu_{p, \epsilon}(A)= \begin{cases}(1-\epsilon) \mathbf{P}(A) & \text { if } A \neq \Omega \\ 1, & \text { if } A=\Omega\end{cases}
$$


For every permutation $\sigma$ of $\Omega$, we can define a probability measure $\mathbf{P}^{\sigma}$ on $\mathcal{F}$, whose value on the singletons (we avoid braces to simplify writing) is

$$
\mathbf{P}^{\sigma}(\sigma(i))=\nu_{p, \epsilon}\left(E_{i}^{\sigma}\right)-\nu_{p, \epsilon}\left(E_{i+1}^{\sigma}\right)
$$

where $E_{i}^{\sigma}=\left\{\sigma(i), \ldots, \sigma\left(2^{T}\right)\right\}$, for all $i \in \Omega$, and $E_{2^{T}+1}^{\sigma}=\emptyset$. It turns out that the class $\mathcal{P}_{p, \epsilon}$ is the convex hull of probabilities $\mathbf{P}^{\sigma}{ }^{\prime}$ s.

We consider a CRRA utility function defined, for $\gamma>0, \gamma \neq 1$, as

$$
u_{\gamma}(x)=\frac{x^{1-\gamma}}{1-\gamma}, \quad \text { for } x>0 .
$$

For every random variable $V_{T} \in \mathbb{R}_{++}^{\Omega}$, we can define the functional

$$
\mathbf{C E U}_{\gamma, p, \epsilon}\left[V_{T}\right]=\oint u_{\gamma}\left(V_{T}\right) \mathrm{d} \nu_{p, \epsilon},
$$

where the integral on the right side is of Choquet type. In particular, since $u_{\gamma}$ is strictly increasing, for all $V_{T} \in \mathbb{R}_{++}^{\Omega}$, if $\sigma$ is a permutation of $\Omega$ such that $V_{T}(\sigma(1)) \leq \ldots \leq V_{T}\left(\sigma\left(2^{T}\right)\right)$ then the functional $\mathbf{C E U _ { \gamma , p , \epsilon }}$ can be expressed (see [8]) as follows

$$
\mathbf{C E U}_{\gamma, p, \epsilon}\left[V_{T}\right]=\sum_{k=1}^{2^{T}} \mathbf{P}^{\sigma}(k) \frac{\left(V_{T}(k)\right)^{1-\gamma}}{1-\gamma} .
$$

Proposition 1. The functional $\mathbf{C E U}_{\gamma, p, \epsilon}$ is concave, that is, for all $V_{T}, V_{T}^{\prime} \in$ $\mathbb{R}_{++}^{\Omega}$ and all $\alpha \in[0,1]$, it holds

$$
\mathbf{C E U}_{\gamma, p, \epsilon}\left[\alpha V_{T}+(1-\alpha) V_{T}^{\prime}\right] \geq \alpha \mathbf{C E U}_{\gamma, p, \epsilon}\left[V_{T}\right]+(1-\alpha) \mathbf{C E U}_{\gamma, p, \epsilon}\left[V_{T}^{\prime}\right] .
$$

Proof. Since $u_{\gamma}$ is (strictly) concave, for all $i \in \Omega$ and $\alpha \in[0,1]$, we have

$$
u_{\gamma}\left(\alpha V_{T}(i)+(1-\alpha) V_{T}^{\prime}(i)\right) \geq \alpha u_{\gamma}\left(V_{T}(i)\right)+(1-\alpha) u_{\gamma}\left(V_{T}^{\prime}(i)\right) .
$$

Hence, since $\nu_{p, \epsilon}$ is completely monotone and so 2-monotone, Theorems 4.24 and 4.35 in [8] imply (by monotonicity and concavity of the Choquet integral)

$$
\begin{aligned}
& \mathbf{C E U}_{\gamma, p, \epsilon}\left[\alpha V_{T}+(1-\alpha) V_{T}^{\prime}\right]=\oint u_{\gamma}\left(\alpha V_{T}+(1-\alpha) V_{T}^{\prime}\right) \mathrm{d} \nu_{p, \epsilon} \\
& \geq \oint\left[\alpha u_{\gamma}\left(V_{T}\right)+(1-\alpha) u_{\gamma}\left(V_{T}^{\prime}\right)\right] \mathrm{d} \nu_{p, \epsilon} \\
& \geq \alpha \oint u_{\gamma}\left(V_{T}\right) \mathrm{d} \nu_{p, \epsilon}+(1-\alpha) \oint u_{\gamma}\left(V_{T}^{\prime}\right) \mathrm{d} \nu_{p, \epsilon} \\
& =\alpha \mathbf{C E U}_{\gamma, p, \epsilon}\left[V_{T}\right]+(1-\alpha) \mathbf{C E U}_{\gamma, p, \epsilon}\left[V_{T}^{\prime}\right] \text {. }
\end{aligned}
$$

By Theorem 4.39 in [8], we also have that

$$
\mathbf{C E U}_{\gamma, p, \epsilon}\left[V_{T}\right]=\min _{\mathbf{P}^{\prime} \in \mathcal{P}_{p, \epsilon}} \mathbf{E}^{\mathbf{P}^{\prime}}\left[u_{\gamma}\left(V_{T}\right)\right]
$$

therefore, $\mathbf{C E U}_{\gamma, p, \epsilon}$ is a lower expected utility and maximizing it we are actually applying a maximin criterion of choice. 
Given an initial wealth $V_{0}>0$, our aim is to select a self-financing strategy $\left\{\theta_{0}, \ldots, \theta_{T-1}\right\}$ resulting in a final wealth $V_{T} \in \mathbb{R}_{++}^{\Omega}$, solving

$$
\max _{\theta_{0}, \ldots, \theta_{T-1}} \mathbf{C E U}_{\gamma, p, \epsilon}\left[V_{T}\right]
$$

Taking into account the completeness of the market, the above problem can be rewritten maximizing over the final wealth $V_{T}$

$$
\begin{gathered}
\begin{array}{c}
\text { maximize } \mathbf{C E U _ { \gamma , p , \epsilon }}\left[V_{T}\right] \\
\text { subject to: }
\end{array} \\
\left\{\begin{array}{l}
\mathbf{E}^{\mathbf{Q}}\left[V_{T}\right]-(1+r)^{T} V_{0}=0, \\
V_{T} \in \mathbb{R}_{++}^{\Omega} .
\end{array}\right.
\end{gathered}
$$

By Proposition 1, the objective function in (3) is a concave function on $\mathbb{R}_{++}^{\Omega}$, subject to a linear constraint, therefore every local maximum is a global maximum and, further, the set of global maxima is convex [1].

Since by (2) the computation of $\mathbf{C E U}_{\gamma, p, \epsilon}\left[V_{T}\right]$ depends on a permutation $\sigma$ such that the values of $V_{T}$ are increasingly ordered, the above problem (3) can be decomposed in a family of optimization problems, each indexed by a permutation $\sigma$ of $\Omega=\left\{1, \ldots, 2^{T}\right\}$, corresponding to a possible ordering of $V_{T}$ :

$$
\begin{gathered}
\text { maximize }\left[\sum_{k=1}^{2^{T}} \mathbf{P}^{\sigma}(k) \frac{\left(V_{T}(k)\right)^{1-\gamma}}{1-\gamma}\right] \\
\text { subject to: } \\
\left\{\begin{array}{l}
\sum_{k=1}^{2^{T}} \mathbf{Q}(k) V_{T}(k)-(1+r)^{T} V_{0}=0, \\
V_{T}(\sigma(i-1))-V_{T}(\sigma(i)) \leq 0, \quad \text { for all } i \in \Omega \backslash\{1\}, \\
V_{T} \in \mathbb{R}_{++}^{\Omega} .
\end{array}\right.
\end{gathered}
$$

Denoting by $V_{T}^{\sigma}$ an optimal solution related to permutation $\sigma$, an optimal solution $V_{T}^{*}$ for (3) can be found by selecting $V_{T}^{\sigma}$ for a permutation $\sigma$ where the objective function is maximum. Notice that there can be more permutations where the maximum is attained.

Proposition 2. The following statements hold:

(i) for every permutation $\sigma$, problem (4) has an optimal solution and such optimal solution is unique;

(ii) problem (3) has an optimal solution and such optimal solution is unique.

Proof. (i). The subset $\mathcal{V}^{\sigma}$ of $\mathbb{R}^{\Omega}$ satisfying the equality and inequality constraints in (4) is closed, and $\mathcal{V}^{\sigma} \cap \mathbb{R}_{++}^{\Omega} \neq \emptyset$, since $V_{T}=(1+r)^{T} V_{0} \in \mathcal{V}^{\sigma} \cap \mathbb{R}_{++}^{\Omega}$. Proceeding as in the proof of Theorem 2.12 in [13], we have that problem (4) has an optimal solution $V_{T}^{\sigma}$, and such optimal solution is unique since the objective function in (4) is strictly concave [1], as $u_{\gamma}$ is strictly concave. 
(ii). Statement (i) implies that problem (3) has an optimal solution $V_{T}^{*}$ obtained by selecting $V_{T}^{\sigma}$ for a permutation $\sigma$ where the objective function is maximum. Suppose there are two permutations $\sigma, \sigma^{\prime}$ attaining the maximum and such that $V_{T}^{\sigma} \neq V_{T}^{\sigma^{\prime}}$. Since the set of optimal solutions of (3) is convex, this implies that, for all $\alpha \in[0,1], V_{T}^{\alpha}=\alpha V_{T}^{\sigma}+(1-\alpha) V_{T}^{\sigma^{\prime}}$ is an optimal solution of (3). So, since the number of permutations of $\Omega$ is finite, we can find an $\alpha^{*}$ such that $V_{T}^{\alpha^{*}}$ solves (4) for a permutation $\sigma^{\prime \prime}$ but $V_{T}^{\alpha^{*}} \neq V_{T}^{\sigma^{\prime \prime}}$, reaching a contradiction. Finally, this implies that problem (3) has a unique optimal solution.

\subsection{Characterization of the Case $p=\frac{1}{2}$}

Here we focus on the special case $p=\frac{1}{2}$, for which the probability distribution $\mathbf{P}^{\sigma}$ on $\Omega$ is such that

$$
\mathbf{P}^{\sigma}(\sigma(1))=\frac{1+\left(2^{T}-1\right) \epsilon}{2^{T}} \quad \text { and } \quad \mathbf{P}^{\sigma}(\sigma(i))=\frac{1-\epsilon}{2^{T}}, \quad \text { for all } i \in \Omega \backslash\{1\} .
$$

The definition of $\mathbf{P}^{\sigma}$ shows that only the value $\sigma(1)$ of every permutation $\sigma$ deserves attention: all the permutations $\sigma, \sigma^{\prime}$ such that $\sigma(1)=\sigma^{\prime}(1)$ can be considered to be equivalent. Therefore, we can restrict to $2^{T}$ arbitrary permutations $\sigma_{1}, \ldots, \sigma_{2^{T}}$ satisfying $\sigma_{h}(1)=h$, for all $h \in \Omega$.

In this case, the family of optimization problems (4) can be reduced to a family of optimization problems indexed by the permutations $\sigma_{1}, \ldots, \sigma_{2^{T}}$ :

$$
\begin{gathered}
\text { maximize }\left[\sum_{k=1}^{2^{T}} \mathbf{P}^{\sigma_{h}}(k) \frac{\left(V_{T}(k)\right)^{1-\gamma}}{1-\gamma}\right] \\
\text { subject to: } \\
\left\{\begin{array}{l}
\sum_{k=1}^{2^{T}} \mathbf{Q}(k) V_{T}(k)-(1+r)^{T} V_{0}=0, \\
V_{T}\left(\sigma_{h}(1)\right)-V_{T}\left(\sigma_{h}(i)\right) \leq 0, \quad \text { for all } i \in \Omega \backslash\{1\}, \\
V_{T} \in \mathbb{R}_{++}^{\Omega} .
\end{array}\right.
\end{gathered}
$$

Analogously to problem (4), for every permutation $\sigma_{h}$, problem (5) has a unique optimal solution $V_{T}^{\sigma_{h}}$. Then, the optimal solution $V_{T}^{*}$ for (3) can be found by selecting a $V_{T}^{\sigma_{h}}$ for a permutation $\sigma_{h}$ (possibly non-unique) where the objective function is maximum. This shows that, when $p=\frac{1}{2}$, problem (3) can be solved by solving $2^{T}$ problems (5).

Below we provide a characterization of the optimal solution of problem (3). In what follows, let $\sigma$ be a permutation of $\Omega$ and $I \subseteq \Omega \backslash\{1\}$. If $I \neq \emptyset$, for all $i \in I$, consider the constants

$$
\begin{array}{r}
A_{i}^{\sigma, I}=\frac{\mathbf{P}^{\sigma}(\sigma(i))}{\sum_{k \in I \cup\{1\}} \mathbf{P}^{\sigma}(\sigma(k))}\left[\left(\frac{\mathbf{Q}(\sigma(i))}{\mathbf{P}^{\sigma}(\sigma(i))}-\frac{\mathbf{Q}(\sigma(1))}{\mathbf{P}^{\sigma}(\sigma(1))}\right)\left(\sum_{k \in(I \cup\{1\}) \backslash\{i\}} \mathbf{P}^{\sigma}(\sigma(k))\right)\right. \\
\left.+\sum_{k \in I \backslash\{i\}}\left(\mathbf{P}^{\sigma}(\sigma(k))\left(\frac{\mathbf{Q}(\sigma(1))}{\mathbf{P}^{\sigma}(\sigma(1))}-\frac{\mathbf{Q}(\sigma(k))}{\mathbf{P}^{\sigma}(\sigma(k))}\right)\right)\right],
\end{array}
$$


where summations over an empty set are intended to be 0 .

In turn, the above constants are used to define the following weights:

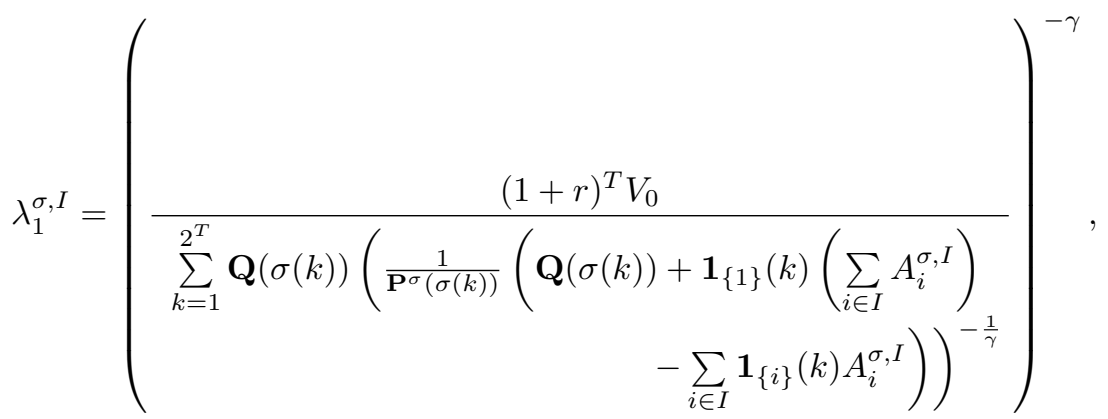

where $\mathbf{1}_{\{i\}}$ is the indicator function of the singleton $\{i\}$, while, for all $i \in I$, set

$$
\lambda_{i}^{\sigma, I}=A_{i}^{\sigma, I} \lambda_{1}^{\sigma, I},
$$

and $\lambda_{i}^{\sigma, I}=0$, for all $i \in \Omega \backslash(I \cup\{1\})$. Notice that, if $I=\emptyset$, then the two inner summations involving $I$ in the definition of $\lambda_{1}^{\sigma, I}$ are set to 0 : in this case, only $\lambda_{1}^{\sigma, I}$ will be non-null.

Theorem 1. For $p=\frac{1}{2}$ and $T \geq 1$, a random variable $V_{T} \in \mathbb{R}_{++}^{\Omega}$ is the optimal solution of problem (3) if and only if there is a permutation $\sigma$ of $\Omega$ and a subset $I \subseteq \Omega \backslash\{1\}$ inducing the weights $\lambda_{i}^{\sigma, I}$ 's such that the following conditions hold:

(i) $\lambda_{i}^{\sigma, I} \geq 0$, for all $i \in I$;

(ii) $V_{T}(\sigma(1))=\left(\frac{1}{\mathbf{P}^{\sigma}(\sigma(1))}\left(\mathbf{Q}(\sigma(1)) \lambda_{1}^{\sigma, I}+\sum_{k=2}^{2^{T}} \lambda_{k}^{\sigma, I}\right)\right)^{-\frac{1}{\gamma}}$, for all $i \in \Omega \backslash\{1\}$,

$$
V_{T}(\sigma(i))=\left(\frac{1}{\mathbf{P}^{\sigma}(\sigma(i))}\left(\mathbf{Q}(\sigma(i)) \lambda_{1}^{\sigma, I}-\lambda_{i}^{\sigma, I}\right)\right)^{-\frac{1}{\gamma}}
$$

(iii) $V_{T}(\sigma(1)) \leq V_{T}(\sigma(i))$, for all $i \in \Omega \backslash(I \cup\{1\})$;

and there is no other permutation $\sigma^{\prime}$ of $\Omega$ and no other subset $I^{\prime} \subseteq \Omega \backslash\{1\}$ determining the weights $\lambda^{\sigma^{\prime}, I^{\prime}}$ 's and the random variable $V_{T}^{\prime} \in \mathbb{R}_{++}^{\Omega}$ satisfying (i)-(iii) such that $\mathbf{C E U}_{\gamma, p, \epsilon}\left[V_{T}^{\prime}\right]>\mathbf{C E U}_{\gamma, p, \epsilon}\left[V_{T}\right]$.

Proof. Let $\sigma$ be a permutation of $\Omega$. We first show that $V_{T} \in \mathbb{R}_{++}^{\Omega}$ is the optimal solution of the corresponding problem (5) if and only if there is a subset $I \subseteq \Omega \backslash\{1\}$ inducing weights $\lambda_{i}^{\sigma, I}$ 's satisfying (i)-(iii). Denote by $f\left(V_{T}\right)=f\left(V_{T}(1), \ldots, V_{T}\left(2^{T}\right)\right)$ and $g\left(V_{T}\right)=g\left(V_{T}(1), \ldots, V_{T}\left(2^{T}\right)\right)=0$ the objective function and the equality constraint in (5), which are, respectively, (strictly) concave and linear. We also have that all inequality constraints in (5) are linear, therefore, the Karush-Kuhn-Tucker (KKT) conditions are necessary and sufficient in this case [1]. Define the Lagrangian function

$$
L\left(V_{T}, \lambda_{1}, \ldots, \lambda_{2^{T}}\right)=f\left(V_{T}\right)-\lambda_{1} g\left(V_{T}\right)-\sum_{k=2}^{2^{T}} \lambda_{k}\left(V_{T}(\sigma(1))-V_{T}(\sigma(k))\right),
$$


for which it holds

$$
\begin{aligned}
\frac{\partial L}{\partial V_{T}(\sigma(1))} & =\mathbf{P}^{\sigma}(\sigma(1))\left(V_{T}(\sigma(1))\right)^{-\gamma}-\lambda_{1} \mathbf{Q}(\sigma(1))-\sum_{k=2}^{2^{T}} \lambda_{k}, \\
\frac{\partial L}{\partial V_{T}(\sigma(i))} & =\mathbf{P}^{\sigma}(\sigma(i))\left(V_{T}(\sigma(i))\right)^{-\gamma}-\lambda_{1} \mathbf{Q}(\sigma(i))+\lambda_{i}, \quad \text { for all } i \in \Omega \backslash\{1\} .
\end{aligned}
$$

Imposing the KKT conditions, we look for $V_{T} \in \mathbb{R}_{++}^{\Omega}, \lambda_{1} \in \mathbb{R}, \lambda_{2}, \ldots, \lambda_{2^{T}} \geq 0$ such that $\frac{\partial L}{\partial V_{T}(\sigma(k))}=0$, for all $k \in \Omega, g\left(V_{T}\right)=0, V_{T}(\sigma(1)) \leq V_{T}(\sigma(i))$ and $\lambda_{i}\left(V_{T}(\sigma(1))-V_{T}(\sigma(i))\right)=0$, for all $i \in \Omega \backslash\{1\}$.

By $\frac{\partial L}{\partial V_{T}(\sigma(k))}=0$, for all $k \in \Omega$, we derive

$$
\begin{aligned}
& V_{T}(\sigma(1))=\left(\frac{1}{\mathbf{P}^{\sigma}(\sigma(1))}\left(\lambda_{1} \mathbf{Q}(\sigma(1))+\sum_{k=2}^{2^{T}} \lambda_{k}\right)\right)^{-\frac{1}{\gamma}}, \\
& V_{T}(\sigma(i))=\left(\frac{1}{\mathbf{P}^{\sigma}(\sigma(i))}\left(\lambda_{1} \mathbf{Q}(\sigma(i))-\lambda_{i}\right)\right)^{-\frac{1}{\gamma}}, \quad \text { for all } i \in \Omega \backslash\{1\} .
\end{aligned}
$$

Moreover, by the complementary slackness conditions $\lambda_{i}\left(V_{T}(\sigma(1))-V_{T}(\sigma(i))\right)=$ 0 , for all $i \in \Omega \backslash\{1\}$, there must exist $I \subseteq \Omega \backslash\{1\}$ such that $\lambda_{i}=0$, for all $i \in \Omega \backslash(I \cup\{1\})$, while, for all $i \in I, V_{T}(\sigma(1))-V_{T}(\sigma(i))=0$.

The case $I=\emptyset$ is trivial, thus suppose $I \neq \emptyset$. For every $i \in I$, equation $V_{T}(\sigma(1))-V_{T}(\sigma(i))=0$ holds if and only if

$$
\left(\frac{\mathbf{Q}(\sigma(1))}{\mathbf{P}^{\sigma}(\sigma(1))}-\frac{\mathbf{Q}(\sigma(i))}{\mathbf{P}^{\sigma}(\sigma(i))}\right) \lambda_{1}+\left(\frac{1}{\mathbf{P}^{\sigma}(\sigma(1))}+\frac{1}{\mathbf{P}^{\sigma}(\sigma(i))}\right) \lambda_{i}+\frac{1}{\mathbf{P}^{\sigma}(\sigma(1))} \sum_{k \in I \backslash\{i\}} \lambda_{k}=0 .
$$

Choose an enumeration of $I \cup\{1\}=\left\{i_{1}, i_{2}, \ldots, i_{n}\right\}$ with $i_{1}=1$. Then the above equations give rise to the homogeneous linear system $A \mathbf{x}=\mathbf{0}$, whose unknown is the column vector $\mathbf{x}=\left[\begin{array}{llll}\lambda_{1} & \lambda_{i_{2}} & \cdots & \lambda_{i_{n}}\end{array}\right]^{T} \in \mathbb{R}^{n \times 1}$ and whose coefficient matrix is $A=[\mathbf{q} \mid B] \in \mathbb{R}^{(n-1) \times n}$ where

$$
\begin{aligned}
& \mathbf{q}=\left[\left(\frac{\mathbf{Q}(\sigma(1))}{\mathbf{P}^{\sigma}(\sigma(1))}-\frac{\mathbf{Q}\left(\sigma\left(i_{2}\right)\right)}{\mathbf{P}^{\sigma}\left(\sigma\left(i_{2}\right)\right)}\right)\left(\frac{\mathbf{Q}(\sigma(1))}{\mathbf{P}^{\sigma}(\sigma(1))}-\frac{\mathbf{Q}\left(\sigma\left(i_{3}\right)\right)}{\mathbf{P}^{\sigma}\left(\sigma\left(i_{3}\right)\right)}\right) \cdots\left(\frac{\mathbf{Q}(\sigma(1))}{\mathbf{P}^{\sigma}(\sigma(1))}-\frac{\mathbf{Q}\left(\sigma\left(i_{n}\right)\right)}{\mathbf{P}^{\sigma}\left(\sigma\left(i_{n}\right)\right)}\right)\right]^{T}, \\
& B=\left[\begin{array}{cccc}
\left(\frac{1}{\mathbf{P}^{\sigma}(\sigma(1))}+\frac{1}{\mathbf{P}^{\sigma}\left(\sigma\left(i_{2}\right)\right)}\right) & \frac{1}{\mathbf{P}^{\sigma}(\sigma(1))} & \cdots & \frac{1}{\mathbf{P}^{\sigma}(\sigma(1))} \\
\overline{\mathbf{P}^{\sigma}(\sigma(1))} & \left(\frac{1}{\mathbf{P}^{\sigma}(\sigma(1))}+\frac{1}{\mathbf{P}^{\sigma}\left(\sigma\left(i_{3}\right)\right)}\right) & \cdots & \overline{\mathbf{P}^{\sigma}(\sigma(1))} \\
\vdots & \vdots & \cdots & \vdots \\
\overline{\mathbf{P}^{\sigma}(\sigma(1))} & \overline{\mathbf{P}^{\sigma}(\sigma(1))} & \cdots & \left(\frac{1}{\mathbf{P}^{\sigma}(\sigma(1))}+\frac{1}{\mathbf{P}^{\sigma}\left(\sigma\left(i_{n}\right)\right)}\right)
\end{array}\right] .
\end{aligned}
$$

Hence, $B \in \mathbb{R}^{(n-1) \times(n-1)}$ can be decomposed in the sum of two matrices $C, D \in \mathbb{R}^{(n-1) \times(n-1)}$, where $C$ is a constant matrix with all entries equal to $\frac{1}{\mathbf{P}^{\sigma}(\sigma(1))}$ and $D$ is the diagonal matrix whose diagonal contains the elements 
$\frac{1}{\mathbf{P}^{\sigma}\left(\sigma\left(i_{2}\right)\right)}, \ldots, \frac{1}{\mathbf{P}^{\sigma}\left(\sigma\left(i_{n}\right)\right)}$. Since the determinant does not change subtracting the first row from all other rows, we can consider the matrix

$$
\left[\begin{array}{cccc}
\left(\frac{1}{\mathbf{P}^{\sigma}(\sigma(1))}+\frac{1}{\mathbf{P}^{\sigma}\left(\sigma\left(i_{2}\right)\right)}\right) & \frac{1}{\mathbf{P}^{\sigma}(\sigma(1))} & \cdots & \frac{1}{\mathbf{P}^{\sigma}(\sigma(1))} \\
-\frac{1}{\mathbf{P}^{\sigma}\left(\sigma\left(i_{2}\right)\right)} & \frac{1}{\mathbf{P}^{\sigma}\left(\sigma\left(i_{3}\right)\right)} & \cdots & 0 \\
\vdots & \vdots & \cdots & \vdots \\
-\frac{1}{\mathbf{P}^{\sigma}\left(\sigma\left(i_{2}\right)\right)} & 0 & \cdots & \frac{1}{\mathbf{P}^{\sigma}\left(\sigma\left(i_{n}\right)\right)}
\end{array}\right] .
$$

Applying the Laplace expansion along the first row we get that

$$
\operatorname{det} B=\operatorname{det}(C+D)=\frac{\sum_{j=1}^{n} \mathbf{P}^{\sigma}\left(\sigma\left(i_{j}\right)\right)}{\prod_{j=1}^{n} \mathbf{P}^{\sigma}\left(\sigma\left(i_{j}\right)\right)},
$$

and since $\operatorname{det} B \neq 0$, we have that $\operatorname{rank} B=n-1$ and the system admits non-trivial solutions, depending on one real parameter that we identify with $\lambda_{1}$. Now, apply Cramer's rule to the reduced system $B \mathbf{y}=-\lambda_{1} \mathbf{q}$ with unknown the column vector $\mathbf{y}=\left[\begin{array}{lll}\lambda_{i_{2}} & \cdots & \lambda_{i_{n}}\end{array}\right]^{T} \in \mathbb{R}^{(n-1) \times 1}$.

For $j=2, \ldots, n$, denote by $B_{j-1}$ the matrix obtained by substituting the $(j-$ 1)-th column of $B$ with the vector $-\lambda_{1} \mathbf{q}$. Applying the Laplace expansion along the $(j-1)$-th column of $B_{j-1}$ and noticing that all minors can be transformed (by swapping rows and keeping track of sign changes) in the sum of a constant matrix and a diagonal matrix (possibly with a zero on the diagonal), we have

$$
\begin{aligned}
& \operatorname{det} B_{j-1}=\left[\left(\frac{\mathbf{Q}\left(\sigma\left(i_{j}\right)\right)}{\mathbf{P}^{\sigma}\left(\sigma\left(i_{j}\right)\right)}-\frac{\mathbf{Q}(\sigma(1))}{\mathbf{P}^{\sigma}(\sigma(1))}\right) \frac{\sum_{k \in(I \cup\{1\}) \backslash\left\{i_{j}\right\}} \mathbf{P}^{\sigma}(\sigma(k))}{\prod_{k \in(I \cup\{1\}) \backslash\left\{i_{j}\right\}} \mathbf{P}^{\sigma}(\sigma(k))}\right. \\
& \left.+\sum_{k \in I \backslash\left\{i_{j}\right\}}\left(\left(\frac{\mathbf{Q}(\sigma(1))}{\mathbf{P}^{\sigma}(\sigma(1))}-\frac{\mathbf{Q}(\sigma(k))}{\mathbf{P}^{\sigma}(\sigma(k))}\right) \frac{1}{\prod_{s \in(I \cup\{1\}) \backslash\left\{i_{j}, k\right\}} \mathbf{P}^{\sigma}(\sigma(s))}\right)\right] \lambda_{1}
\end{aligned}
$$

therefore, $\lambda_{i_{j}}=\frac{\operatorname{det} B_{j-1}}{\operatorname{det} B}=A_{i_{j}}^{\sigma, I} \lambda_{1}$.

Substituting in $g\left(V_{T}\right)=0$ the expressions of $V_{T}(\sigma(k))$, for all $k \in \Omega$, and $\lambda_{i}$, for all $i \in \Omega \backslash\{1\}$, we get for $\lambda_{1}$ the expression of $\lambda_{1}^{\sigma, I}$, thus $\lambda_{i}$ coincides with $\lambda_{i}^{\sigma, I}$. Hence, $V_{T} \in \mathbb{R}_{++}^{\Omega}$ is the optimal solution for the problem (5) if and only if there exists $I \subseteq \Omega \backslash\{1\}$ inducing weights $\lambda_{i}^{\sigma, I}$ 's satisfying (i)-(iii).

For every $h \in \Omega$, let $\sigma_{h}$ be a permutation of $\Omega$ such that $\sigma_{h}(1)=h$ and denote by $V_{T}^{\sigma_{h}}$ the optimal solution of problem (5) for $\sigma_{h}$. By the definition of the Choquet integral [8], problem (3) is equivalent to maximizing $\mathbf{C E U}_{\gamma, p, \epsilon}$ over optimal solutions of the family of problems (4), indexed by all permutations of $\Omega$. In turn, since, for every permutations $\sigma, \sigma^{\prime}$ of $\Omega$ such that $\sigma(1)=\sigma^{\prime}(1)$, it holds $\mathbf{P}^{\sigma}=\mathbf{P}^{\sigma^{\prime}}$, such maximization can be reduced to maximizing $\mathbf{C E U _ { \gamma , p , \epsilon }}$ over optimal solutions of the family of problems (5), indexed by permutations $\sigma_{1}, \ldots, \sigma_{2^{T}}$ of $\Omega$. This finally proves the theorem.

Theorem 1 allows to find the analytic expression of the optimal solution of problem (3) in the case $p=\frac{1}{2}$, reducing it to $2^{T}$ combinatorial optimization 
problems, each corresponding to a permutation $\sigma_{h}$ of $\Omega$ such that $\sigma_{h}(1)=h$, for all $h \in \Omega$.

Example 1. Consider a two-period model with $T=2, \gamma=2, \epsilon=\frac{1}{50}, p=\frac{1}{2}$, $u=2$, and $d=\frac{1}{2}$. Suppose $S_{0}=€ 100, V_{0}=€ 10$, and the risk-free interest rate is $r=5 \%$ over every period. This implies that $q=\frac{11}{30}$ while, for every permutation $\sigma$ of $\Omega=\{1,2,3,4\}$, it holds $\mathbf{P}^{\sigma}(\sigma(1))=\frac{53}{200}$ and $\mathbf{P}^{\sigma}(\sigma(i))=\frac{49}{200}$.

For $\sigma_{1}=\langle 1,2,3,4\rangle$ the only subset of $\Omega \backslash\{1\}$ satisfying (i)-(iii) is $I=\{4\}$ with:

- $\lambda_{1}^{\sigma_{1}, I}=0.00822164, \lambda_{2}^{\sigma_{1}, I}=0, \lambda_{3}^{\sigma_{1}, I}=0, \lambda_{4}^{\sigma_{1}, I}=0.00118255$,

- $V_{2}^{\sigma_{1}}(1)=10.7623, V_{2}^{\sigma_{1}}(2)=11.328, V_{2}^{\sigma_{1}}(3)=11.328, V_{2}^{\sigma_{1}}(4)=10.7623$,

- $\mathbf{C E U}_{\gamma, p, \epsilon}\left[V_{2}^{\sigma_{1}}\right]=-0.0906436$.

For $\sigma_{2}=\langle 2,1,3,4\rangle$ the only subset of $\Omega \backslash\{1\}$ satisfying (i)-(iii) is $I=\{4\}$ with:

- $\lambda_{1}^{\sigma_{2}, I}=0.00803641, \lambda_{2}^{\sigma_{2}, I}=0, \lambda_{3}^{\sigma_{2}, I}=0, \lambda_{4}^{\sigma_{2}, I}=0.000778428$,

- $V_{2}^{\sigma_{2}}(1)=15.0585, V_{2}^{\sigma_{2}}(2)=10.0101, V_{2}^{\sigma_{2}}(3)=11.4578, V_{2}^{\sigma_{2}}(4)=10.0101$,

- $\mathbf{C E U}_{\gamma, p, \epsilon}\left[V_{2}^{\sigma_{2}}\right]=-0.0886014$.

For $\sigma_{3}=\langle 3,1,2,4\rangle$ the only subset of $\Omega \backslash\{1\}$ satisfying (i)-(iii) is $I=\{4\}$ with:

- $\lambda_{1}^{\sigma_{3}, I}=0.00803641, \lambda_{2}^{\sigma_{3}, I}=0, \lambda_{3}^{\sigma_{3}, I}=0, \lambda_{4}^{\sigma_{3}, I}=0.000778428$,

- $V_{2}^{\sigma_{3}}(1)=15.0585, V_{2}^{\sigma_{3}}(2)=11.4578, V_{2}^{\sigma_{3}}(3)=10.0101, V_{2}^{\sigma_{3}}(4)=10.0101$,

- $\mathbf{C E U}_{\gamma, p, \epsilon}\left[V_{2}^{\sigma_{3}}\right]=-0.0886014$.

For $\sigma_{4}=\langle 4,1,2,3\rangle$ the only subset of $\Omega \backslash\{1\}$ satisfying (i)-(iii) is $I=\emptyset$ with:

- $\lambda_{1}^{\sigma_{4}, I}=0.0079751, \lambda_{2}^{\sigma_{4}, I}=0, \lambda_{3}^{\sigma_{4}, I}=0, \lambda_{4}^{\sigma_{4}, I}=0$,

- $V_{2}^{\sigma_{4}}(1)=15.1162, V_{2}^{\sigma_{4}}(2)=11.5017, V_{2}^{\sigma_{4}}(3)=11.5017, V_{2}^{\sigma_{4}}(4)=9.1017$,

- $\mathbf{C E U}_{\gamma, p, \epsilon}\left[V_{2}^{\sigma_{4}}\right]=-0.0879255$.

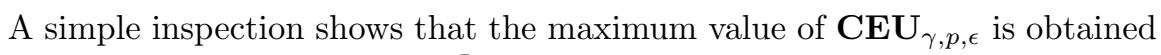
for $\sigma_{4}$, therefore we take $V_{2}^{*}=V_{2}^{\sigma_{4}}$.

Using the martingale property with respect to $\mathbf{Q}$ of the wealth process $\left\{V_{0}^{*}, V_{1}^{*}, V_{2}^{*}\right\}$, that is $V_{1}^{*}=\frac{\mathbf{E}_{1}^{\mathbf{Q}}\left[V_{2}^{*}\right]}{1+r}$ and $V_{0}^{*}=V_{0}=\frac{\mathbf{E}_{0}^{\mathbf{Q}}\left[V_{2}^{*}\right]}{(1+r)^{2}}$, we can recover the optimal self-financing strategy $\left\{\theta_{0}, \theta_{1}\right\}$ through (1):

\begin{tabular}{c|ccc|cc}
$\Omega$ & $V_{0}^{*}$ & $V_{1}^{*}$ & $V_{2}^{*}$ & $\theta_{0}$ & $\theta_{1}$ \\
\hline 1 & $€ 10$ & $€ 12.2162$ & $€ 15.1162$ & 0.018 & 0.012 \\
2 & $€ 10$ & $€ 12.2162$ & $€ 11.5017$ & 0.018 & 0.012 \\
3 & $€ 10$ & $€ 9.5064$ & $€ 11.5017$ & 0.018 & 0.032 \\
4 & $€ 10$ & $€ 9.5064$ & $€ 9.1017$ & 0.018 & 0.032
\end{tabular}




\section{The Effect of $\epsilon$ on the Optimal Portfolio}

Now we investigate the effect of $\epsilon$ on the optimal portfolio. Figures 1 and 2 show the optimal value of $\mathbf{C E U}_{\gamma, p, \epsilon}$ for $V_{0}=€ 10, u \in\{1.2,1.4,1.6,1.8,2\}, d=\frac{1}{u}$, $r=5 \%, \gamma=2, p=\frac{1}{2}$ and $\epsilon$ ranging in $[0,1)$ with step 0.01 , for $T=2$ and $T=3$, respectively. In particular, $\epsilon=0$ stands for absence of ambiguity.

In both figures we can see that, for increasing $\epsilon$, the optimal value of $\mathbf{C E U}_{\gamma, p, \epsilon}$ decreases until reaching a constant value that corresponds to $u_{\gamma}\left((1+r)^{T} V_{0}\right)$. It actually holds that, once the optimal value of $\mathbf{C E U} \mathbf{U}_{\gamma, p, \epsilon}$ reaches $u_{\gamma}\left((1+r)^{T} V_{0}\right)$, the optimal portfolio results in the final risk-free wealth $V_{T}^{*}=(1+r)^{T} V_{0}$. In such cases, the corresponding self-financing strategy is such that $\theta_{t}=0$, for $t=0, \ldots, T-1$, i.e., the optimal portfolio consists of only a risk-free bond investment for all the periods.

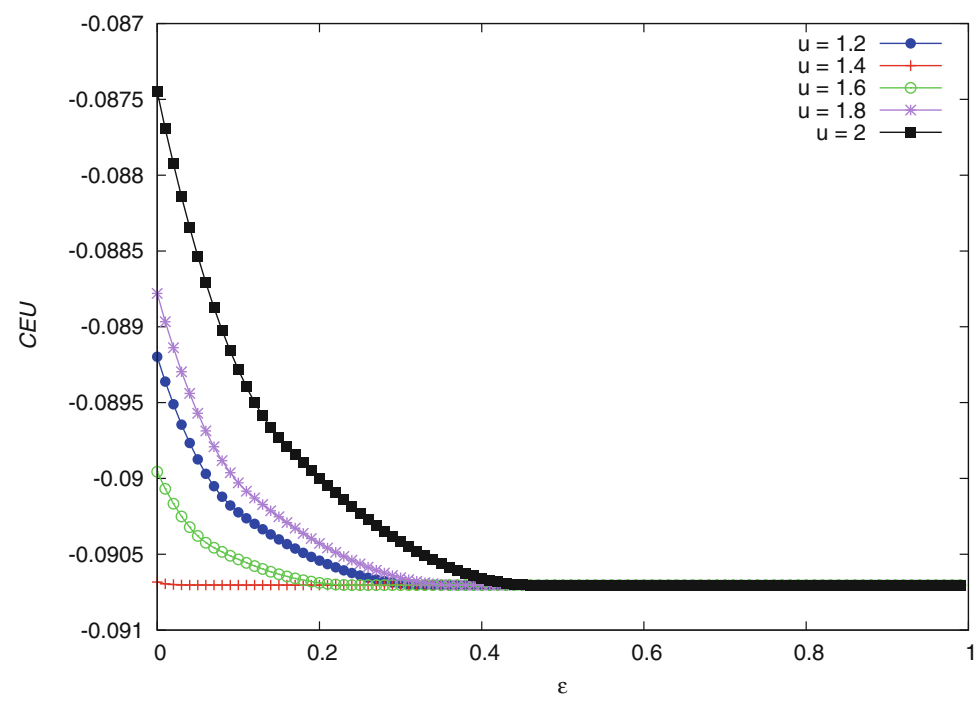

Fig. 1. Optimal values of $\mathbf{C E U}_{\gamma, p, \epsilon}$ for $T=2, V_{0}=€ 10, u \in\{1.2,1.4,1.6,1.8,2\}$, $d=\frac{1}{u}, r=5 \%, \gamma=2, p=\frac{1}{2}$ and $\epsilon$ ranging in [0,1) with step 0.01 .

Figures 1 and 2 highlight the existence of a value $\epsilon^{*}\left(T, \gamma, p, u, d, r, V_{0}\right)$ above which the optimal self-financing strategy reduces to 0 for all times. In a sense, we may think at $\epsilon^{*}\left(T, \gamma, p, u, d, r, V_{0}\right)$ as a threshold above which the ambiguity incorporated in the real-world probability $\mathbf{P}$ through the $\epsilon$-contamination is so high to make the risk-free portfolio the most suitable choice. The following Table 1 reports the intervals containing $\epsilon^{*}\left(T, \gamma, p, u, d, r, V_{0}\right)$ for the parameter setting of Figs. 1 and 2. Let us stress that a numerical approximation of $\epsilon^{*}\left(T, \gamma, p, u, d, r, V_{0}\right)$ can be achieved by applying a suitable bisection algorithm, while its analytic expression will be the aim of future research. 


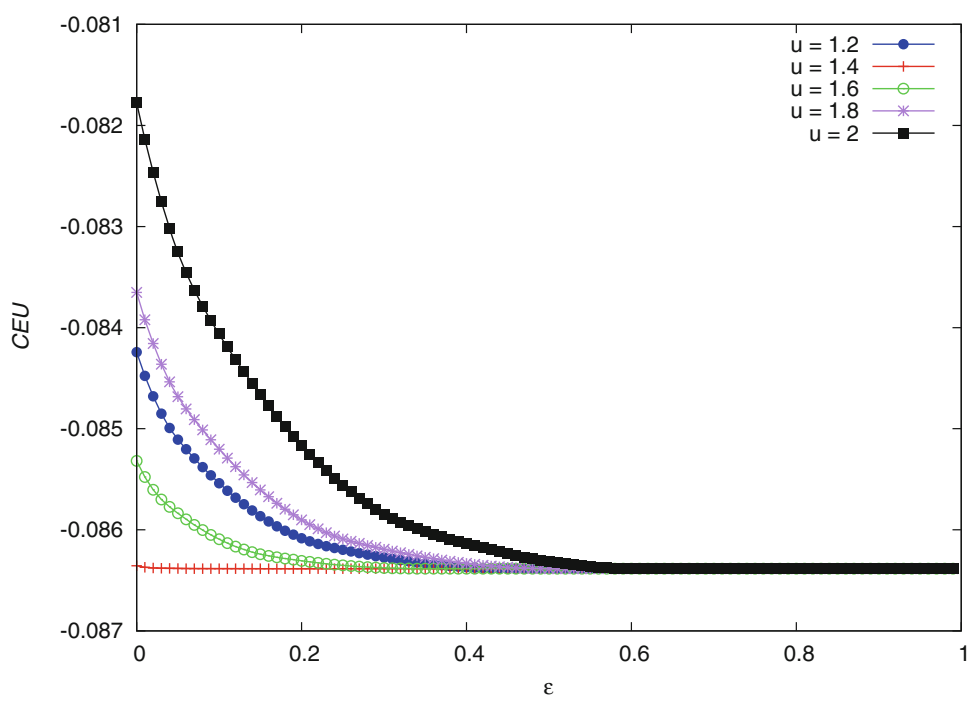

Fig. 2. Optimal values of $\mathbf{C E U}_{\gamma, p, \epsilon}$ for $T=3, V_{0}=€ 10, u \in\{1.2,1.4,1.6,1.8,2\}$, $d=\frac{1}{u}, r=5 \%, \gamma=2, p=\frac{1}{2}$ and $\epsilon$ ranging in $[0,1)$ with step 0.01 .

Table 1. Intervals containing $\epsilon^{*}\left(T, \gamma, p, u, d, r, V_{0}\right)$ for $T \in\{2,3\}, V_{0}=€ 10, u \in$ $\{1.2,1.4,1.6,1.8,2\}, d=\frac{1}{u}, r=5 \%, \gamma=2, p=\frac{1}{2}$.

\begin{tabular}{l|l|l}
\hline$\gamma=2$ & $T=2$ & $T=3$ \\
\hline$u=1.2$ & {$[0.33,0.34]$} & {$[0.45,0.46]$} \\
\hline$u=1.4$ & {$[0.04,0.05]$} & {$[0.06,0.07]$} \\
\hline$u=1.6$ & {$[0.23,0.24]$} & {$[0.33,0.34]$} \\
\hline$u=1.8$ & {$[0.36,0.37]$} & {$[0.49,0.5]$} \\
\hline$u=2$ & {$[0.46,0.47]$} & {$[0.6,0.61]$} \\
\hline
\end{tabular}

\section{Conclusions and Future Works}

In this paper we formulate a dynamic portfolio selection problem under ambiguity by referring to an $\epsilon$-contaminated binomial market model and a CRRA utility function. We provide a characterization of the optimal solution in the case the stock price returns are uniformly distributed and investigate the effect of the contamination parameter $\epsilon$ on the optimal portfolio. It turns out that, in the uniform case, one can find a threshold for $\epsilon$ above which the optimal portfolio reduces to a risk-free investment on every period. An analytic characterization of such threshold is reserved for future research. Further, a characterization of the optimal solution in the general case remains open, as well as the generalization to other classes of utility functions. Another line of investigation is the design of efficient algorithms to solve the family of concave optimization problems to which the initial 
problem can be reduced, since, being indexed by the permutations of the sample space, they constitute a computational challenge.

\section{References}

1. Boyd, S., Vandenberghe, L.: Convex Optimization. Cambridge University Press, Cambridge (2004)

2. Coletti, G., Petturiti, D., Vantaggi, B.: Rationality principles for preferences on belief functions. Kybernetika 51(3), 486-507 (2015)

3. Cox, J., Ross, S., Rubinstein, M.: Option pricing: a simplified approach. J. Finan. Econ. 7(3), 229-263 (1979)

4. Ellsberg, D.: Risk, ambiguity, and the savage axioms. Q. J. Econ. 75(4), 643-669 (1961)

5. Etner, J., Jeleva, M., Tallon, J.M.: Decision theory under ambiguity. J. Econ. Surv. 26(2), 234-270 (2012)

6. Gilboa, I., Marinacci, M.: Ambiguity and the Bayesian paradigm. In: Arló-Costa, H., Hendricks, V.F., van Benthem, J. (eds.) Readings in Formal Epistemology. SGTP, vol. 1, pp. 385-439. Springer, Cham (2016). https://doi.org/10.1007/9783-319-20451-2_21

7. Gilboa, I., Schmeidler, D.: Maxmin expected utility with non-unique prior. J. Math. Econ. 18(2), 141-153 (1989)

8. Grabisch, M.: Set Functions, Games and Capacities in Decision Making. Theory and Decision Library C. Springer, Heidelberg (2016). https://doi.org/10.1007/9783-319-30690-2

9. Huber, P.J.: Robust Statistics. Wiley, New York (1981)

10. Jaffray, J.Y.: Linear utility theory for belief functions. Oper. Res. Lett. 8(2), 107-112 (1989)

11. Jin, H., Yu Zhou, X.: Behavioral portfolio selection in continuous time. Math. Finan. 18(3), 385-426 (2008)

12. Kast, R., Lapied, A., Roubaud, D.: Modelling under ambiguity with dynamically consistent Choquet random walks and Choquet-Brownian motions. Econ. Model. 38, 495-503 (2014)

13. Pascucci, A., Runggaldier, W.: Financial Mathematics: Theory and Problems for Multi-period Models. Springer, Heidelberg (2009). https://doi.org/10.1007/97888-470-2538-7

14. Petturiti, D., Vantaggi, B.: Modeling agent's conditional preferences under objective ambiguity in Dempster-Shafer theory. Int. J. Approximate Reasoning 119, 151-176 (2020)

15. Pflug, G., Wozabal, D.: Ambiguity in portfolio selection. Quant. Finan. 7(4), 435-442 (2007)

16. Pflug, G.C., Pohl, M.: A review on ambiguity in stochastic portfolio optimization. Set-Valued Variational Anal. 26(4), 733-757 (2018)

17. Schmeidler, D.: Subjective probability and expected utility without additivity. Econometrica 57(3), 571-587 (1989)

18. Cerný, A.: Mathematical Techniques in Finance: Tools for Incomplete Markets, 2nd edn. Princeton University Press, Princeton (2009)

19. Walley, P.: Statistical Reasoning with Imprecise Probabilities. Chapman and Hall, London (1991) 